\title{
A Review of Challenges in Display Technology
}

\author{
Askari Mohammad Bagher ${ }^{1}$, Mirzaei Mahmoud Abadi Vahid ${ }^{2}$, Mirhabibi Mohsen ${ }^{1}$ \\ ${ }^{1}$ Department of Physics, Payame Noor University, Tehran, Iran \\ ${ }^{2}$ Department of Physics, Shahid Bahonar University of Kerman, Kerman, Iran
}

Email address:

mb_askari@yahoo.com (A. M. Bagher)

\section{To cite this article:}

Askari Mohammad Bagher, Mirzaei Mahmoud Abadi Vahid, Mirhabibi Mohsen. A Review of Challenges in Display Technology. International Journal of Electrical Components and Energy Conversion. Vol. 3, No. 2, 2017, pp. 26-39. doi: 10.11648/j.ijecec.20170302.12

Received: January 26, 2017; Accepted: February 13, 2017; Published: May 19, 2017

\begin{abstract}
Fast-moving tech is redefining the electronic display as screens get bigger and better. Electrically operated display devices have developed from electromechanical systems for display of text, up to all-electronic devices capable of full-motion 3D color graphic displays. Electromagnetic devices, using a solenoid coil to control a visible flag or flap, were the earliest type, and were used for text displays such as stock market prices and arrival/departure display times. In this article we will discuss about the different types of displays and also about the advantages and disadvantages different types of display technology.
\end{abstract}

Keywords: Display Technology, Electronic Devices, Electromagnetic Devices

\section{Introduction}

The cathode ray tube was the workhorse of text and video display technology for several decades until being displaced by plasma, liquid crystal (LCD) and solid-state devices. With the advent of microprocessors and microelectronic devices, many more individual picture elements (pixels) could be incorporated into one display device, allowing graphic displays and video. In this article, we will review the most types of displays.

\section{Basic CRT structure and operation}

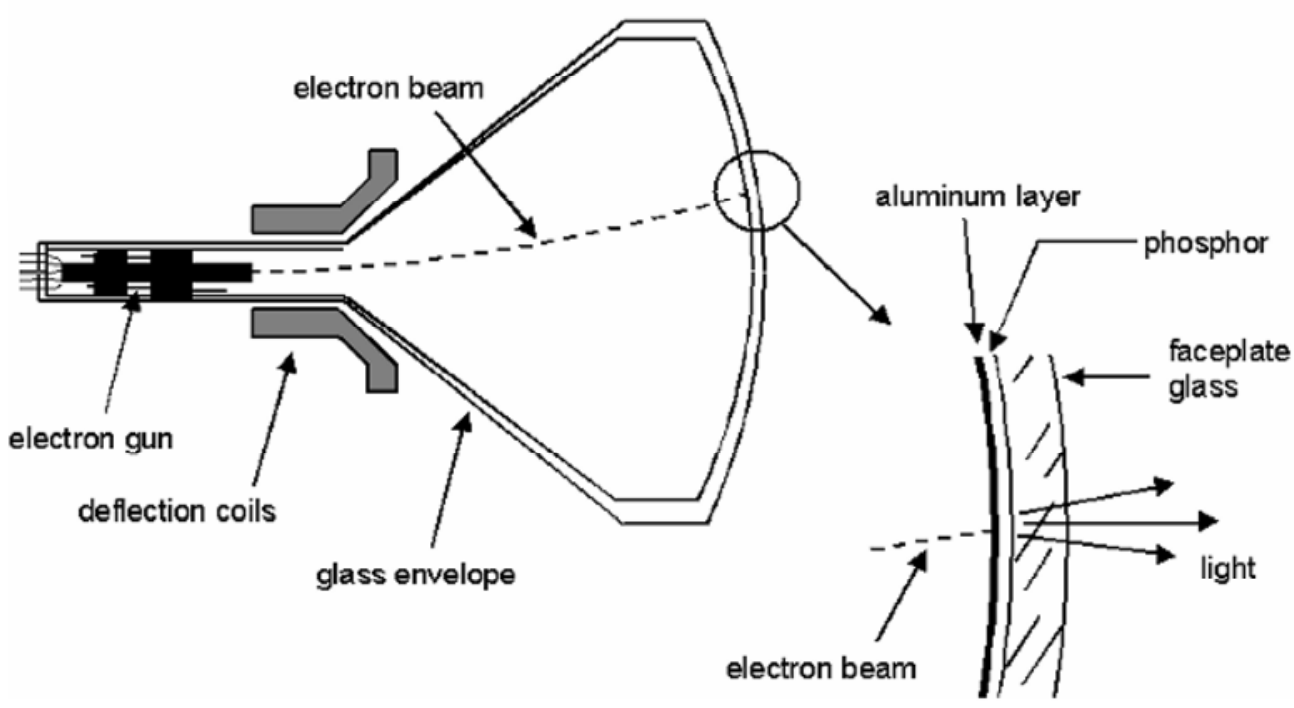

Figure 1. CRT structure (http://what-when-how.com). 


\section{Cathode Ray Tube Display (CRT)}

The cathode ray tube (CRT) is a vacuum tube that contains one or more electron guns and a phosphorescent screen, and is used to display images. [1] It modulates, accelerates, and deflects electron beam (s) onto the screen to create the images. The images may represent electrical waveforms (oscilloscope), pictures (television, computer monitor), radar targets, or others. CRTs have also been used as memory devices, in which case the visible light emitted from the fluorescent material (if any) is not intended to have significant meaning to a visual observer. The CRT uses an evacuated glass envelope which is large, deep, fairly heavy, and relatively fragile. As a matter of safety, the face is typically made of thick lead glass so as to be highly shatterresistant and to block most X-ray emissions, particularly if the CRT is used in a consumer product. Newer display technologies can also be made in larger sizes; whereas 38" to 40" was about the largest size of a CRT TV. In television sets and computer monitors, the entire front area of the tube is scanned repetitively and systematically in a fixed pattern called a raster. An image is produced by controlling the intensity of each of the three electron beams, one for each additive primary color (red, green, and blue) with a video signal as a reference. In all modern CRT monitors and televisions, the beams are bent by magnetic deflection, a varying magnetic field generated by coils and driven by electronic circuits around the neck of the tube, although electrostatic deflection is commonly used in oscilloscopes, a type of electronic test instrument. [2] Figure 1 shows CRT structure.

\subsection{Cathode Ray Tube Display Advantage}

(1) Resolution and Aspect Ratio: They operate at any resolution, geometry and aspect ratio without the need for rescaling the image.

(2) Highest Resolutions: CRTs run at the highest pixel resolutions generally available.

(3) Black-Level and Contrast: Produce a very dark black and the highest contrast levels normally available. Suitable for use even in dimly lit or dark environments.

(4) Color and Gray-Scale Accuracy: CRTs produce the very best color and gray-scale and are the reference standard for all professional calibrations. They have a perfectly smooth gray-scale with an infinite number of intensity levels. Other display technologies are expected to reproduce the natural power-law Gamma curve of a CRT, but can only do so approximately.

(5) Motion Artifacts: CRTs have fast response times and no motion artifacts. Best for rapidly moving or changing images.

(6) Cost: CRTs are less expensive than comparable displays using other display technologies.

\subsection{Cathode Ray Tube Display Disadvantage}

(1) Sharpness: The CRT's Gaussian beam profile produces images with softer edges that are not as sharp as an LCD at its native resolution. Imperfect focus and color registration also reduce sharpness. Generally sharper than LCDs at other than native resolutions.

(2) Interference: All color CRTs produce annoying Moiré patterns. Many monitors include Moiré reduction, which normally doesn't eliminate the Moiré interference patterns entirely.

(3) Geometric Distortion: Subject to geometric distortion and screen regulation problems. Also affected by magnetic fields from other equipment including other CRTs.

(4) Brightness: Relatively bright but not as bright as LCDs. Not suitable for very brightly lit environments.

(5) Screen Shape: Some CRTs have a rounded spherical or cylindrical shape screen. Newer CRTs are flat.

(6) Emissions: CRTs give off electric, magnetic and electromagnetic fields. There is considerable controversy as to whether any of these pose a health hazard, particularly magnetic fields. The most authoritative scientific studies conclude that they are not harmful but some people remain unconvinced.

(7) Physical: They are large, heavy, and bulky. They consume a lot of electricity and produce a lot of heat. [3]

\section{Light-Emitting Diode Display (LED)}

A light-emitting diode (LED) is a two-lead semiconductor light source. It is a $\mathrm{p}-\mathrm{n}$ junction diode, which emits light when activated.[4] When a suitable voltage is applied to the leads, electrons are able to recombine with electron holes within the device, releasing energy in the form of photons. This effect is called electroluminescence, and the color of the light is determined by the energy band gap of the semiconductor. An LED is often small in area (less than 1 $\mathrm{mm} 2$ ) and integrated optical components may be used to shape its radiation pattern.[5] Appearing as practical electronic components in 1962, the earliest LEDs emitted low-intensity infrared light. Infrared LEDs are still frequently used as transmitting elements in remote-control circuits, such as those in remote controls for a wide variety of consumer electronics. The first visible-light LEDs were also of low intensity and limited to red. Modern LEDs are available across the visible, ultraviolet, and infrared wavelengths, with very high brightness. Figure 2 shows LED structure:

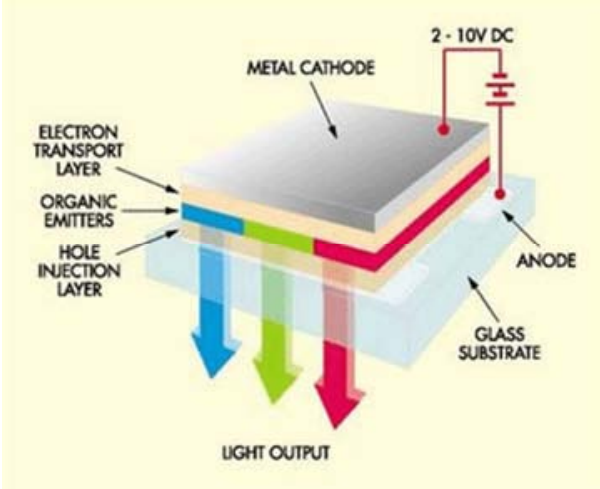

Figure 2. LED structure (www.ledke.com). 


\subsection{Light-Emitting Diode Display Advantages}

(1) Efficiency: LEDs emit more lumens per watt than incandescent light bulbs.

(2) Color: LEDs can emit light of an intended color without using any color filters as traditional lighting methods need.

(3) Size: LEDs can be very small.

(4) Warmup time: LEDs light up very quickly. A typical red indicator LED will achieve full brightness in under a microsecond.

(5) Cycling: LEDs are ideal for uses subject to frequent on-off cycling, unlike incandescent and fluorescent lamps that fail faster when cycled often, or high-intensity discharge lamps (HID lamps) that require a long time before restarting.

(6) Dimming: LEDs can very easily be dimmed either by pulse-width modulation or lowering the forward current.

(7) Cool light: In contrast to most light sources, LEDs radiate very little heat in the form of IR that can cause damage to sensitive objects or fabrics.

(8) Slow failure: LEDs mostly fail by dimming over time, rather than the abrupt failure of incandescent bulbs.

(9) Lifetime: LEDs can have a relatively long useful life. One report estimates 35,000 to 50,000 hours of useful life, though time to complete failure may be longer.

(10) Shock resistance: LEDs, being solid-state components, are difficult to damage with external shock, unlike fluorescent and incandescent bulbs, which are fragile.

(11) Focus: The solid package of the LED can be designed to focus its light.[6-8]

\subsection{Light-Emitting Diode Display Disadvantages}

(1) Initial price: LEDs are currently slightly more expensive

(2) Temperature dependence: LED performance largely depends on the ambient temperature of the operating environment - or thermal management properties.
(3) Voltage sensitivity: LEDs must be supplied with a voltage above their threshold voltage and a current below their rating. Current and lifetime change greatly with a small change in applied voltage.

(4) Color rendition: Most cool-white LEDs have spectra that differ significantly from a black body radiator like the sun or an incandescent light.

(5) Area light source: Single LEDs do not approximate a point source of light giving a spherical light distribution, but rather a lambertian distribution. So LEDs are difficult to apply to uses needing a spherical light field; however, different fields of light can be manipulated by the application of different optics or "lenses". LEDs cannot provide divergence below a few degrees. In contrast, lasers can. [912].

\section{Electroluminescent Display (ELD)}

Electroluminescent Displays are a type of Flat panel display created by sandwiching a layer of electroluminescent material such as GaAs between two layers of conductors. When current flows, the layer of material emits radiation in the form of visible light. Electroluminescence is an optical and electrical phenomenon where a material emits light in response to an electric current passed through it, or to a strong electric field. EL works by exciting atoms by passing an electric current through them, causing them to emit photons. By varying the material being excited, the colour of the light emitted can be changed. The actual ELD is constructed using flat, opaque electrode strips running parallel to each other, covered by a layer of electroluminescent material, followed by another layer of electrodes, running perpendicular to the bottom layer. This top layer must be transparent in order to let light escape. At each intersection, the material lights, creating a pixel. [13] Figure 3 shows ELD structure.

\section{Monochrome EL Display Structure}

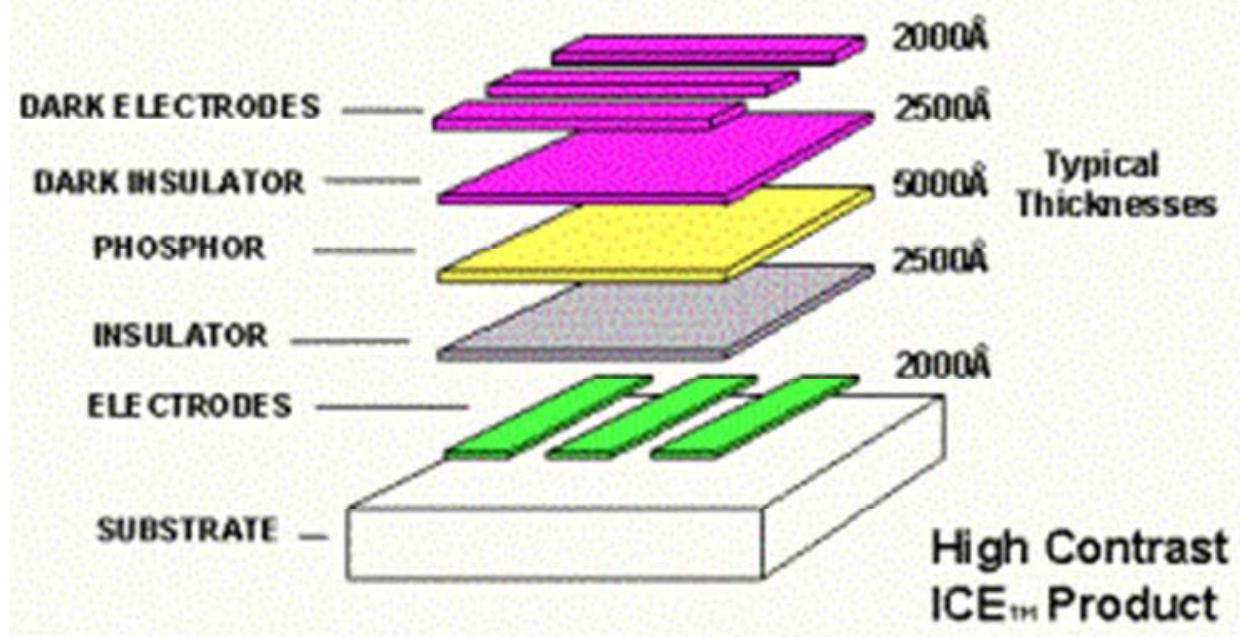

Figure 3. ELD structure. (Indiana University Bloomington). 


\subsection{Electroluminescent Display Advantage}

Electroluminescence has several advantages that has led its being used so widely in electronic devices. Electroluminescence requires very little current in order to work. This is advantageous in small electronic devices because they often run on batteries that are 1.5 Volts or smaller. Electroluminescent devices can produce any color and are very simply designed. [14]

\subsection{Electroluminescent Display Disadvantage}

Though electroluminescence can be advantageous in most cases, it does have a few disadvantages. For example, electroluminescent devices require very little current, but high amounts of voltage - usually between 60 and 600 Volts. These voltages can be consistently provided for electroluminescent devices that are connected to a power line at any time of day. In battery operated, hand-held devices however, a converter circuit that is built into the device must provide the voltage. [14]

\section{Electronic Paper Display}

Electronic paper and e-paper are display devices that mimic the appearance of ordinary ink on paper. [15] Unlike conventional backlit flat panel displays that emit light, electronic paper displays reflect light like paper. This may make them more comfortable to read, and provide a wider viewing angle than most light-emitting displays. The contrast ratio in electronic displays available as of 2008 approaches newspaper, and newly developed displays are slightly better. An ideal e-paper display can be read in direct sunlight without the image appearing to fade. Many electronic paper technologies hold static text and images indefinitely without electricity. Flexible electronic paper uses plastic substrates and plastic electronics for the display backplane. There is ongoing competition among manufacturers to provide fullcolor ability. Applications of electronic visual displays include electronic pricing labels in retail shops and digital signage, time tables at bus stations, electronic billboards, [16] mobile phone displays, and e-readers able to display digital versions of books and magazines.

Low power consumption: One of the advantages of electrophoretic display and electronic ink display is that it consumes less power. Note that this display technology is a bistable display. This means that the image on an electrophoretic display screen is retained even when all power sources are removed. The display is only consuming power whenever the displayed image changes. Figure 4 shows Electronic paper display structure.

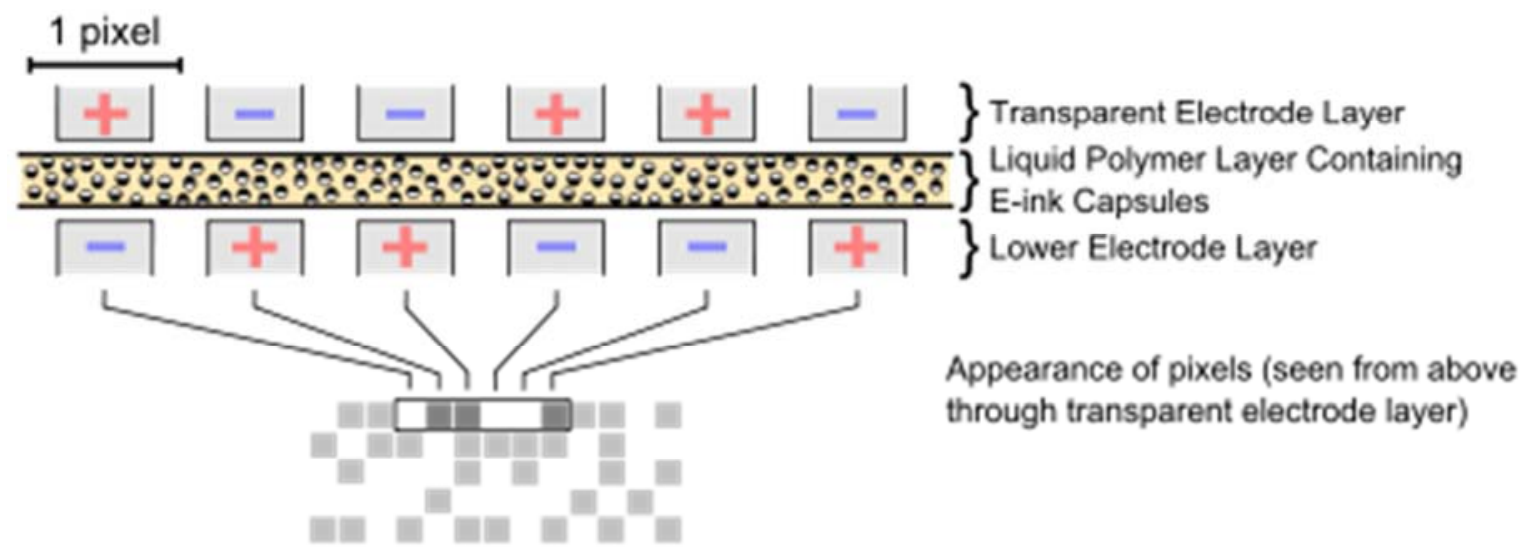

Figure 4. Electronic paper display structure (Wikipedia).

\subsection{Electronic Paper Display Advantage}

(1) This low power consumption makes electrophoretic display and electronic ink display makes suitable for electronic readers and for second displays in smartphones or other consumer electronic devices.

(2) Reflective display: An electrophoretic display or electronic ink display is a type of a reflective display. Take note that liquid crystal display technologies such as in-plane switching or IPS technology and most twisted nematic or TN display screens are emissive display. They require a backlight or internal light source to make the displayed image brighter to the users.

(3) Same readability as print: Another advantage of electrophoretic display and electronic ink display is that the images produced are similar with printed materials such as newspapers. This is because these displays use the same pigment or ink commonly used in print media.

(4) Same LCD monitor resolution: Current electrophoretic displays can support a pixel density of 170 pixels per inch. This is similar to most LCD monitors. However, compared to LCD, electrophoretic display and electronic paper display have better readability in direct sunlight. It also has wider viewing angles than standard LCD monitors-especially TN displays.

(5) Thin and flexible displays: The components of electrophoretic or electronic ink display can be made thin and flexible due to advancements in the technology behind polymer transistors. [17] 


\subsection{Electronic Paper Display Disadvantage}

(1) Very low refresh rate: A notable disadvantage of electrophoretic display and electronic ink display is that it has very low refresh rate when compared to LCD IPS technology and AMOLED display technology. This is also true for other electronic paper display technologies.

(2) Ghosting effect: Electrophoretic display and electronic ink display are prone to ghosting effect. A shadow of an image may be visible after refreshing parts of the screen. This ghosting effect is due to the low refresh rate and the fact that the display technology works by moving charged pigment particles. Some particles end up stuck in the visible surface of a microcapsule.

(3) High market competition: Another disadvantage of electrophoretic display and electronic ink display is that it competes with existing display technologies that have become industry standards. Note that IPS displays and AMOLED displays have become very popular in smartphones and tablet computers, as well as in large screens such as televisions and computer monitors.
(4) Still needs backlighting: One of the advantages of reflective displays is their reliance on ambient light. This means less power consumption form backlighting. However, this advantage can also be a disadvantage for electrophoretic display and electronic ink display. [17]

\section{Plasma Display Panel (PDP)}

A plasma display panel (PDP) is a type of flat panel display often used for large television displays. Many tiny cells located between two panels of glass hold an inert mixture of noble gases (neon and xenon). The gas in the cells is electrically turned into a plasma, which then excites phosphors to emit light. Plasma display technology offers the advantages of producing displays with large, very thin screens and bright images with wide viewing angles. With the advent of "seamless" plasma display technology, it has become possible to display more than one image on the video wall simultaneously, to adjust color balance, and to switch between contents from multiple inputs in the video wall. [1819]. Figure 5 shows Plasma display panel structure

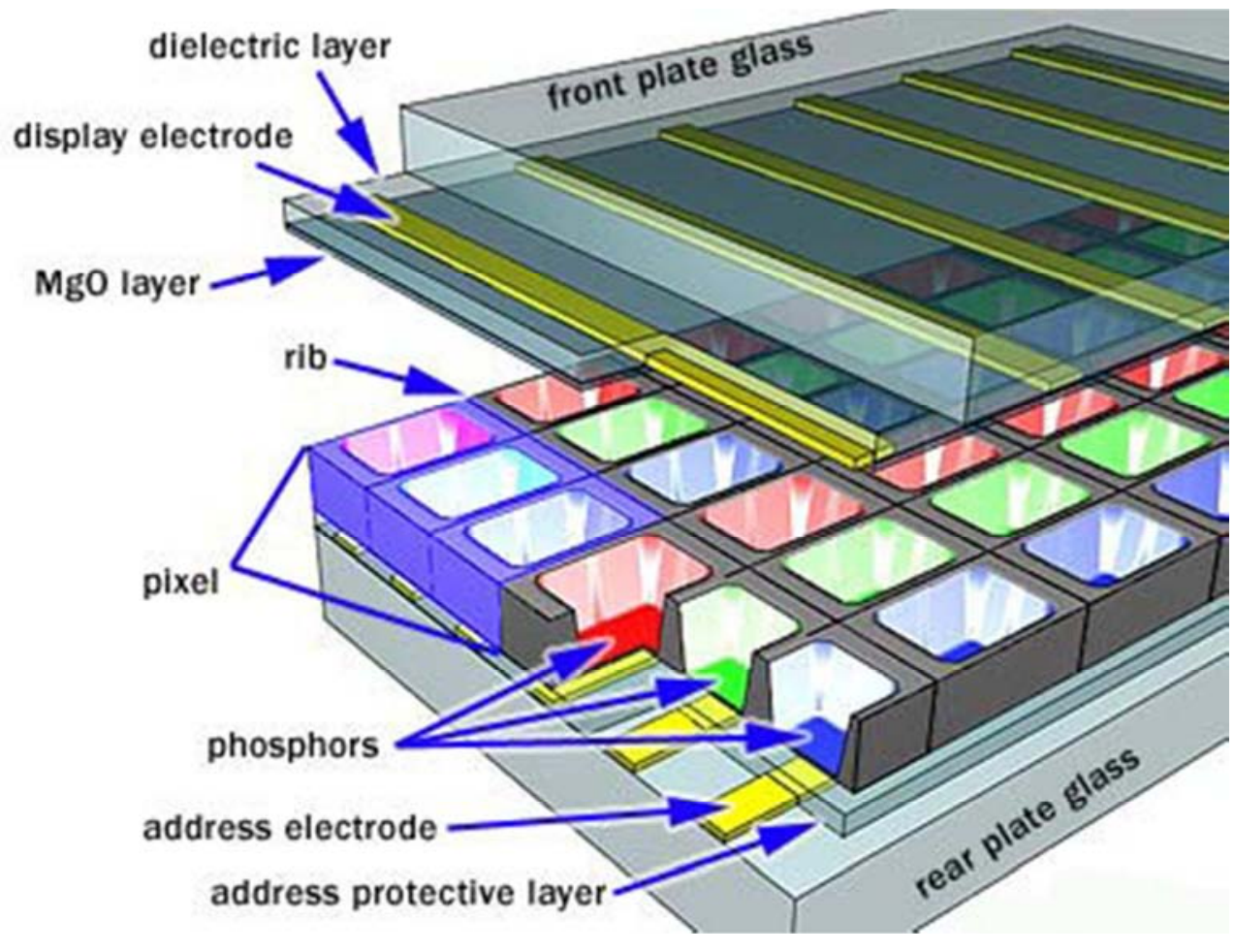

Figure 5. Plasma display panel structure (http://www.practical-home-theater-guide.com/plasma-display.html).

\subsection{Plasma Display Panel Advantages}

(1) Capable of producing deeper blacks allowing for superior contrast ratio

(2) Wider viewing angles than those of LCD; images do not suffer from degradation at less than straight ahead angles like LCDs.

(3) Less visible motion blur, thanks in large part to very high refresh rates and a faster response time, contributing to superior performance when displaying content with significant amounts of rapid motion.

(4) Superior uniformity. LCD panel backlights nearly always produce uneven brightness levels, although this is not always noticeable. High-end computer monitors have technologies to try to compensate for the uniformity problem.

(5) Unaffected by clouding from the polishing process. Some LCD panel types, like IPS, require a polishing process that can introduce a haze usually referred to as "clouding".

(6) Less expensive for the buyer per square inch than LCD, particularly when equivalent performance is considered. [13] [20] 


\subsection{Plasma Display Panel Disadvantages}

(1) Earlier generation displays were more susceptible to screen burn-in and image retention. Recent models have a pixel orbiter that moves the entire picture slower than is noticeable to the human eye, which reduces the effect of burn-in but does not prevent it.

(2) Due to the bistable nature of the colour and intensity generating method, some people will notice that plasma displays have a shimmering or flickering effect with a number of hues, intensities and dither patterns.

(3) Earlier generation displays had phosphors that lost luminosity over time, resulting in gradual decline of absolute image brightness. Newer models have advertised lifespans exceeding 100000 hours, far longer than older CRTs

(4) Uses more electrical power, on average, than an LCD TV using an LED backlight. Older CCFL backlights for LCD panels used quite a bit more power, and older plasma TVs used quite a bit more power than recent models.

(5) Does not work as well at high altitudes above 6,500 feet $(2,000$ metres $)$ due to pressure differential between the gases inside the screen and the air pressure at altitude. It may cause a buzzing noise. Manufacturers rate their screens to indicate the altitude parameters.

(6) For those who wish to listen to AM radio, or are amateur radio operators (hams) or shortwave listeners (SWL), the radio frequency interference (RFI) from these devices can be irritating or disabling.
(7) Plasma displays are generally heavier than LCD, and may require more careful handling such as being kept upright. [21]

\section{Liquid Crystal Display (LCD)}

crystals. Liquid crystals do not emit light directly. [22] LCDs are available to display arbitrary images or fixed images with low information content, which can be displayed or hidden, such as preset words, digits, and 7-segment displays, as in a digital clock. They use the same basic technology, except that arbitrary images are made up of a large number of small pixels, while other displays have larger elements. LCDs are used in a wide range of applications including computer monitors, televisions, instrument panels, aircraft cockpit displays, and indoor and outdoor signage. Small LCD screens are common in portable consumer devices such as digital cameras, watches, calculators, and mobile telephones, including smartphones. LCD screens are also used on consumer electronics products such as DVD players, video game devices and clocks. LCD screens have replaced heavy, bulky cathode ray tube (CRT) displays in nearly all applications. LCD screens are available in a wider range of screen sizes than CRT and plasma displays, with LCD screens available in sizes ranging from tiny digital watches to huge, big-screen television set. Figure 6 shows Liquid crystal display structure.

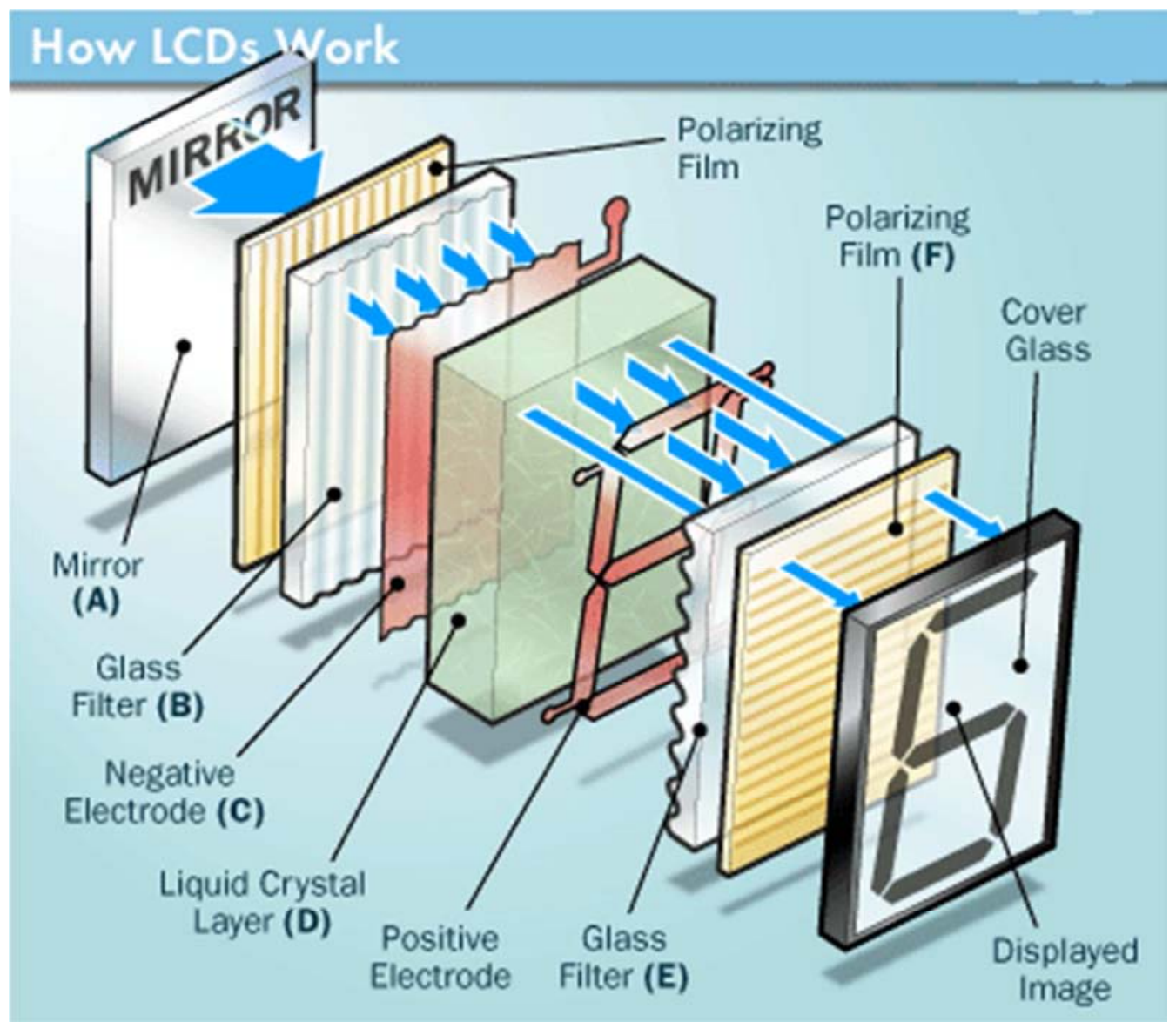

Figure 6. Liquid crystal display structure (Circuits Today). 


\subsection{Liquid Crystal Display Advantage}

(1) Sharpness: Image is perfectly sharp at the native resolution of the panel. LCDs using an analog input require careful adjustment of pixel tracking/phase (see Interference, below).

(2) Geometric Distortion: Zero geometric distortion at the native resolution of the panel. Minor distortion for other resolutions because the images must be rescaled.

(3) Brightness: High peak intensity produces very bright images. Best for brightly lit environments.

(4) Screen Shape: Screens are perfectly flat.

(5) Physical: Thin, with a small footprint. Consume little electricity and produce little heat. [23]

\subsection{Liquid Crystal Display Disadvantage}

(1) Resolution: Each panel has a fixed pixel resolution format determined at the time of manufacture that cannot be changed. All other image resolutions require rescaling, which generally results in significant image degradation, particularly for fine text and graphics. For most applications should only be used at the native resolution of the panel. If you need fine text and graphics at more than one resolution do not get an LCD display.

(2) Interference: LCDs using an analog input require careful adjustment of pixel tracking/phase in order to reduce or eliminate digital noise in the image. Automatic pixel tracking/phase controls seldom produce the optimum setting. Timing drift and jitter may require frequent readjustments during the day. For some displays and video boards you may not be able to entirely eliminate the digital noise.

(3) Viewing Angle: Limited viewing angle. Brightness, contrast, gamma and color mixtures vary with the viewing angle. Can lead to contrast and color reversal at large angles. Need to be viewed as close to straight ahead as possible.

(4) Black-Level, Contrast and Color Saturation: LCDs have difficulty producing black and very dark grays. As a result they generally have lower contrast than CRTs and the color saturation for low intensity colors is also reduced. Not suitable for use in dimly lit and dark environments.

(5) White Saturation: The bright-end of the LCD intensity scale is easily overloaded, which leads to saturation and compression. When this happens the maximum brightness occurs before reaching the peak of the gray-scale or the brightness increases slowly near the maximum. Requires careful adjustment of the Contrast control.

(6) Color and Gray-Scale Accuracy: The internal Gamma and gray-scale of an LCD is very irregular. Special circuitry attempts to fix it, often with only limited success. LCDs typically produce fewer than 256 discrete intensity levels. For some LCDs portions of the gray-scale may be dithered. Images are pleasing but not accurate because of problems with black-level, gray-scale and Gamma, which affects the accuracy of the gray-scale and color mixtures. Generally not suitable for professional image color balancing.

(7) Bad Pixels and Screen Uniformity: LCDs can have many weak or stuck pixels, which are permanently on or off. Some pixels may be improperly connected to adjoining pixels, rows or columns. Also, the panel may not be uniformly illuminated by the backlight resulting in uneven intensity and shading over the screen.

(8) Motion Artifacts: Slow response times and scan rate conversion result in severe motion artifacts and image degradation for moving or rapidly changing images.

(9) Aspect Ratio: LCDs have a fixed resolution and aspect ratio. For panels with a resolution of $1280 \times 1024$ the aspect ratio is $5: 4=1.25$, which is noticeably smaller than the 4 : $3=1.33$ aspect ratio for almost all other standard display modes. For some applications may require switching to a letterboxed 1280x960, which has a 4:3 aspect ratio.

(10) Cost: Considerably more expensive than comparable CRTs. [23]

\section{High-Performance Addressing Display (HPA)}

HPA was primarily designed to provide better image/screen display than the traditional LCD display systems. HPA screens are known to have better contrast and a faster response rate. An HPA-powered LCD screen can display up to 16 million colors. It analyzes each incoming video signal received from the computer and refreshes it with the highest frequency possible, ensuring better display. Although an HPA passive matrix display system doesn't provide a better display than the active-matrix display, it is cheaper thanks to its internal circuitry architecture. [24] [25]

\section{Thin-Film Transistor Display (TFT)}

A thin-film transistor (TFT) is a field-effect transistor that is built by layering thin films on a glass substrate. This differs from the traditional diffusion into a silicon substrate more common for integrated circuits such as microprocessors. The TFT in LCDs controls each individual pixel in the display, as determined by the display driver's integrated circuits. The TFT sets the level of electric field across a liquid crystal capacitor (three per pixel) which controls the polarization of the liquid crystal material. The extent of the liquid crystal polarization determines the amount of light that reaches the color filter. The speed at which the TFT switch effect can be updated for changes in the picture is referred to as the refresh rate, measured in changes per second as Hertz (Hz). [26] [27]. Figure 7 shows Thin-film transistor display structure. 


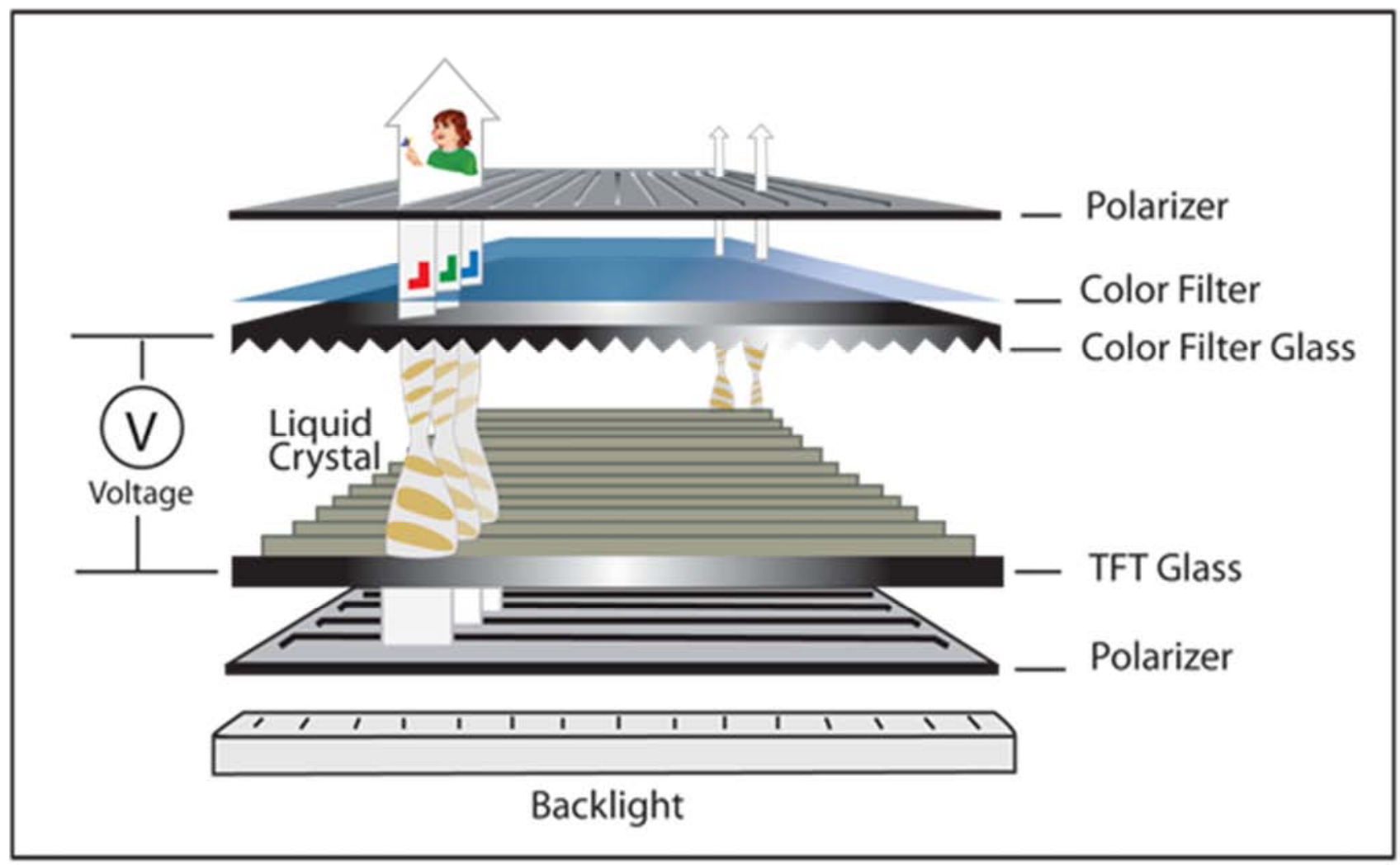

Figure 7. Thin-film transistor display structure (Newhaven Display International, Inc).

\subsection{Thin-Film Transistor Display Advantage}

(1) Sharpness of picture. TFT displays are known for crisp and vibrant colors. This is because instead of having images projected as with CRT monitors - which can cause blurring a TFT display has thousands of individual light sources.

(2) Environmentally conscious power use. TFT displays can use up to half of the power of a traditional monitor. This is a huge energy and money saver, particularly for large offices that require the use of a lot of monitors.

(3) Weight, size, and overall ease of handling. The significantly reduced size and weight of a TFT monitor means that it can be carried and situated onto a desk far, far easier than older, bulkier CRT monitors.

\subsection{Thin-Film Transistor Display Disadvantage}

(1) Viewing angles. While recent developments have made this issue far less significant than it was even just a few years ago, viewing a TFT monitor from an awkward angle will still result in a distorted or less-than-perfect image.

(2) Static resolution. This means that the resolution of a TFT monitor typically cannot be changed. Fortunately most new monitors of this type offer high definition displays.

(3) Accuracy of colors displayed. LCD monitors have difficulty being properly "tuned" for color, particularly strong blacks and bright whites. For example, it may take some tweaking on behalf of the user before a TFT monitor will accurately display the spectrum of colors on an image as they will be printed out. [28]

\section{Organic Light-Emitting Diode Display (OLED)}

OLEDs are solid-state devices composed of thin films of organic molecules that create light with the application of electricity. OLEDs can provide brighter, crisper displays on electronic devices and use less power than conventional light-emitting diodes (LEDs) or liquid crystal displays (LCDs) used today. OLEDs are made by placing thin films of organic (carbon based) materials between two conductors. When electrical current is applied, a bright light is emitted. The OLED materials emit light and do not require a backlight (unlike LCDs). Each pixel is a small light-emitting diode, in fact. OLEDs emit light they do not require a backlight and so are thinner and more efficient than LCD displays (which do require a white backlight). [29] Figure 8 shows Organic lightemitting diode display structure. 

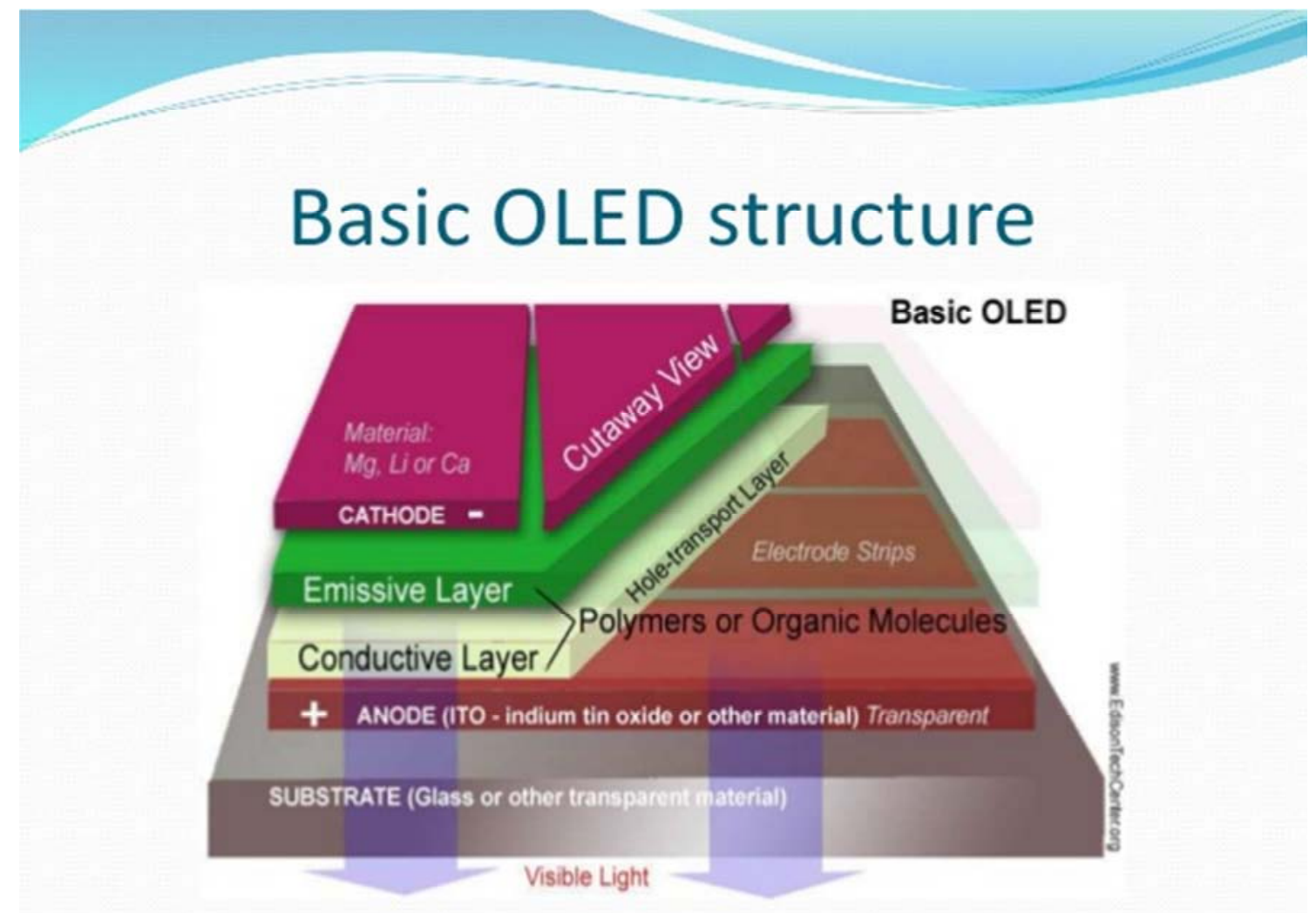

Figure 8. Organic light-emitting diode display structure (www.slideshare.net).

\subsection{Organic Light-Emitting Diode Advantage}

(1) The plastic, organic layers of an OLED are thinner, lighter and more flexible than the crystalline layers in an LED.

(2) Because the light-emitting layers of an OLED are lighter, the substrate of an OLED can be flexible instead of rigid. OLED substrates can be plastic rather than the glass used for LEDs.

(3) OLEDs are brighter than LEDs. Because the organic layers of an OLED are much thinner than the corresponding inorganic crystal layers of an LED, the conductive and emissive layers of an OLED can be multi-layered. Also, LEDs and LCDs require glass for support, and glass absorbs some light. OLEDs do not require glass.

(4) OLEDs do not require backlighting like LCDs. LCDs work by selectively blocking areas of the backlight to make the images that you sees, while OLEDs generate light themselves. Because OLEDs do not require backlighting, they consume much less power than LCDs. This is especially important for battery-operated devices such as cell phones.

(5) OLEDs are easier to produce and can be made to larger sizes. Because OLEDs are essentially plastics, they can be made into large, thin sheets. It is much more difficult to grow and lay down so many liquid crystals.

(6) OLEDs have large fields of view, about 170 degrees. Because LCDs work by blocking light, they have an inherent viewing obstacle from certain angles. OLEDs produce their own light, so they have a much wider viewing range. [30]

\subsection{Organic Light-Emitting Diode Disadvantage}

(1) Usually only with a lifespan of 5,000 hours; 10,000 hours lower than LCD at least.

(2) Large quantity production of large-size screens is not available. It is therefore, only applied to those portable digital products.

(3) Problems of color purity still remains: it is difficult to display fresh and rich colors.

(4) Water can easily damage OLED.

(5) Sunlight Effect: Another disadvantage of OLED display is that they are hard to see in direct sunlight. So if you have open lobbies where sunlight reaches directly, you will not get benefit of viewing these screens.

(6) Manufacturing - Manufacturing processes are expensive right now. [30]

\section{Surface-Conduction Electron-Emitter Display (SED)}

A surface-conduction electron-emitter display (SED) is a display technology for flat panel displays developed by a number of companies. SEDs use nanoscopic-scale electron emitters to energize colored phosphors and produce an image. In a general sense, an SED consists of a matrix of tiny cathode ray tubes, each "tube" forming a single sub-pixel on the screen, grouped in threes to form red-green-blue (RGB) pixels. SEDs combine the advantages of CRTs, namely their 
high contrast ratios, wide viewing angles and very fast response times, with the packaging advantages of LCD and other flat panel displays. They also use much less power than an LCD television of the same size.

After considerable time and effort in the early and mid2000s, SED efforts started winding down in 2009 as LCD became the dominant technology. In August 2010, Canon announced they were shutting down their joint effort to develop SEDs commercially, signalling the end of development efforts. [31] SEDs are closely related to another developing display technology, the field emission display, or FED, differing primarily in the details of the electron emitters. Sony, the main backer of FED, has similarly backed off from their development efforts. [32]

The SED consists of an array of electron emitters and a layer of phosphor, separated by a small space from which all the air has been evacuated. There is a dedicated electron emitter for each pixel in the display. The SED requires no electron-beam focusing, and operates at a much lower voltage than a CRT. The brightness and contrast compare favorably with high-end CRTs. Prototype electron emitters have been developed with diameters of a few nanometers (billionths of a meter), which could yield improved resolution. [33]

\section{Field Emission Display (FED)}

A field emission display (FED) is a flat panel display technology that uses large-area field electron emission sources to provide electrons that strike colored phosphor to produce a color image. In a general sense, an FED consists of a matrix of cathode ray tubes, each tube producing a single sub-pixel, grouped in threes to form red-green-blue (RGB) pixels. FEDs combine the advantages of CRTs, namely their high contrast levels and very fast response times, with the packaging advantages of LCD and other flat panel technologies. They also offer the possibility of requiring less power, about half that of an LCD system. Sony was the major proponent of the FED design and put considerable research and development effort into the system during the 2000s. Sony's FED efforts started winding down in 2009 as LCD became the dominant flat panel technology.[34] In January 2010, AU Optronics announced that it acquired essential FED assets from Sony and intends to continue development of the technology. [35] As of 2016, no large-scale commercial FED production has been undertaken. Figure 9 shows Field emission display structure.

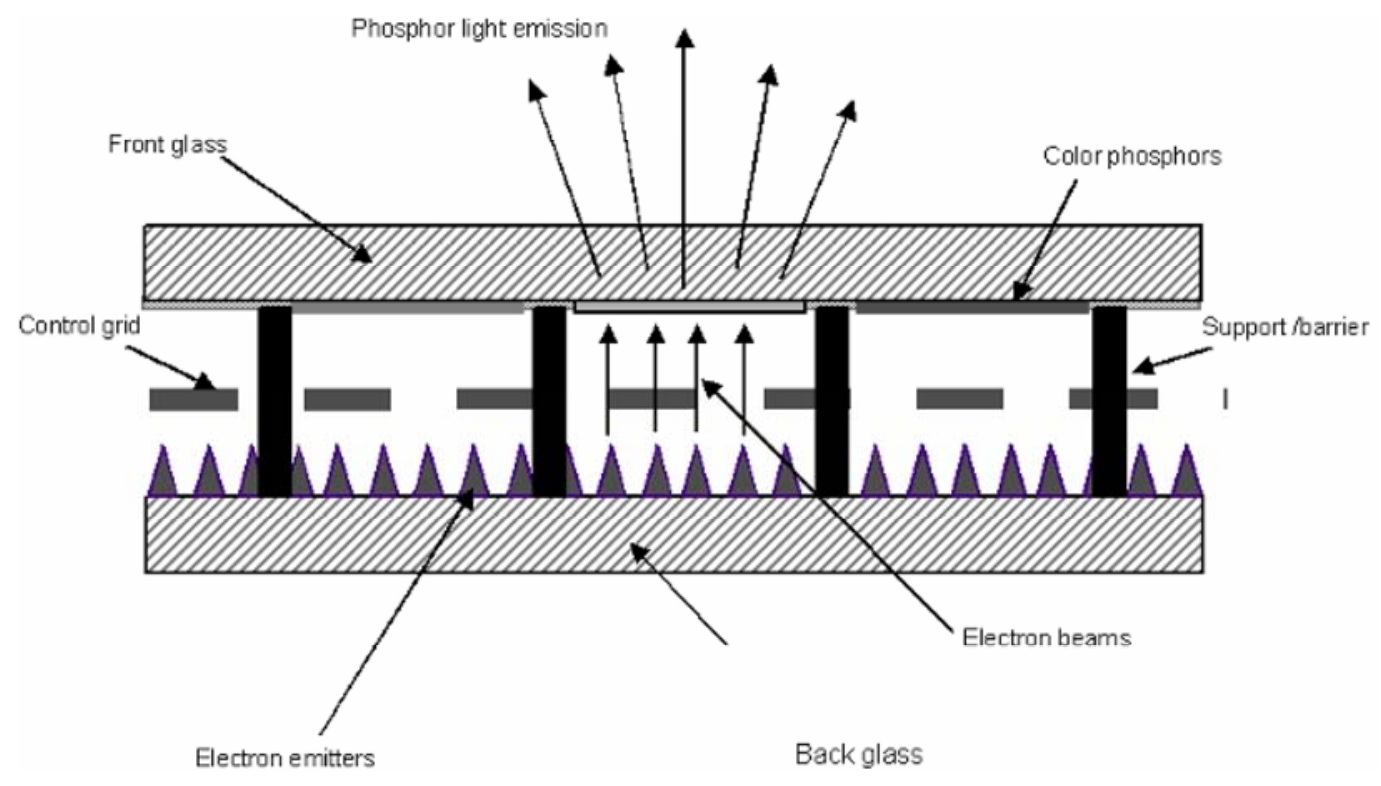

Figure 9. Field emission display structure (In Depth Tutorials and Information).

\subsection{Field Emission Display Fed Advantages}

(1) Full (160 degree) viewing angles horizontally and vertically.

(2) Full motion video without artifacts or contrast loss (20 microsecond response time).

(3) $-45 \mathrm{C}$ to $+85 \mathrm{C}$ operating temperature.

(4) Instant-on at any operating temperature.

(5) High power efficiency.

(6) Costs at or below AMLCD

(7) Very high brightness possible. [36]

\subsection{Field Emission Display Fed Disadvantages}

(1) New, not yet proven technology.

(2) 20 to 85 volt drivers are larger, impacting miniaturization.

(3) Life-times $(10,000 \mathrm{hrs})$ below some competitive technologies [37]

\section{Laser TV}

Laser color television (in short, Laser TV), or Laser color video display utilizes two or more individually modulated 
optical (laser) rays of different colors to produce a combined spot that is scanned and projected across the image plane by a polygon-mirror system or less effectively by optoelectronic means to produce a color-television display. The systems work either by scanning the entire picture a dot at a time and modulating the laser directly at high frequency, much like the electron beams in a cathode ray tube, or by optically spreading and then modulating the laser and scanning a line at a time, the line itself being modulated in much the same way as with Digital Light Processing (DLP). The special case of one ray reduces the system to a monochromatic display as, for example, in black-and-white television. This principle applies to a display as well as to a (front or rear) projection technique with lasers (a laser video projector). [38]

Laser TV Advantage

(1) Be half the weight and cost of plasma displays

(2) Be very thin like plasma and LCD displays are today

(3) Have a very wide color gamut, twice that of current HDTVs

(4) Have a very long life

(5) Maintain full power output for the lifespan of the laser, resulting in a picture that doesn't progressively degrade over time (which happens with technologies such as plasma, LCD and CRT)
(6) Never suffer from screen burn-in

(7) Have a very low power consumption

(8) Have a very thin frame

\section{Quantum Dot Display}

A quantum dot display is an experimental type of display technology. Quantum dots (QD) or semiconductor nanocrystals could provide an alternative for commercial applications such as display technology. This display technology would be similar to organic light-emitting diode (OLED) displays, in that light would be supplied on demand, which would enable more efficient displays. Quantum dots could support large, flexible displays and would not degrade as readily as OLEDs, theoretically making them good candidates for flat-panel TV screens, digital cameras, mobile phones and personal gaming equipment. [39] [40] [41] At present, they are used only to filter light from LEDs to backlit LCDs, rather than as actual displays. Properties and performance are determined by the size and/or composition of the QD. QDs are both photo-active (photoluminescent) and electro-active (electroluminescent) allowing them to be readily incorporated into new emissive display architectures. [42] Figure 10 shows Quantum dot display structure.

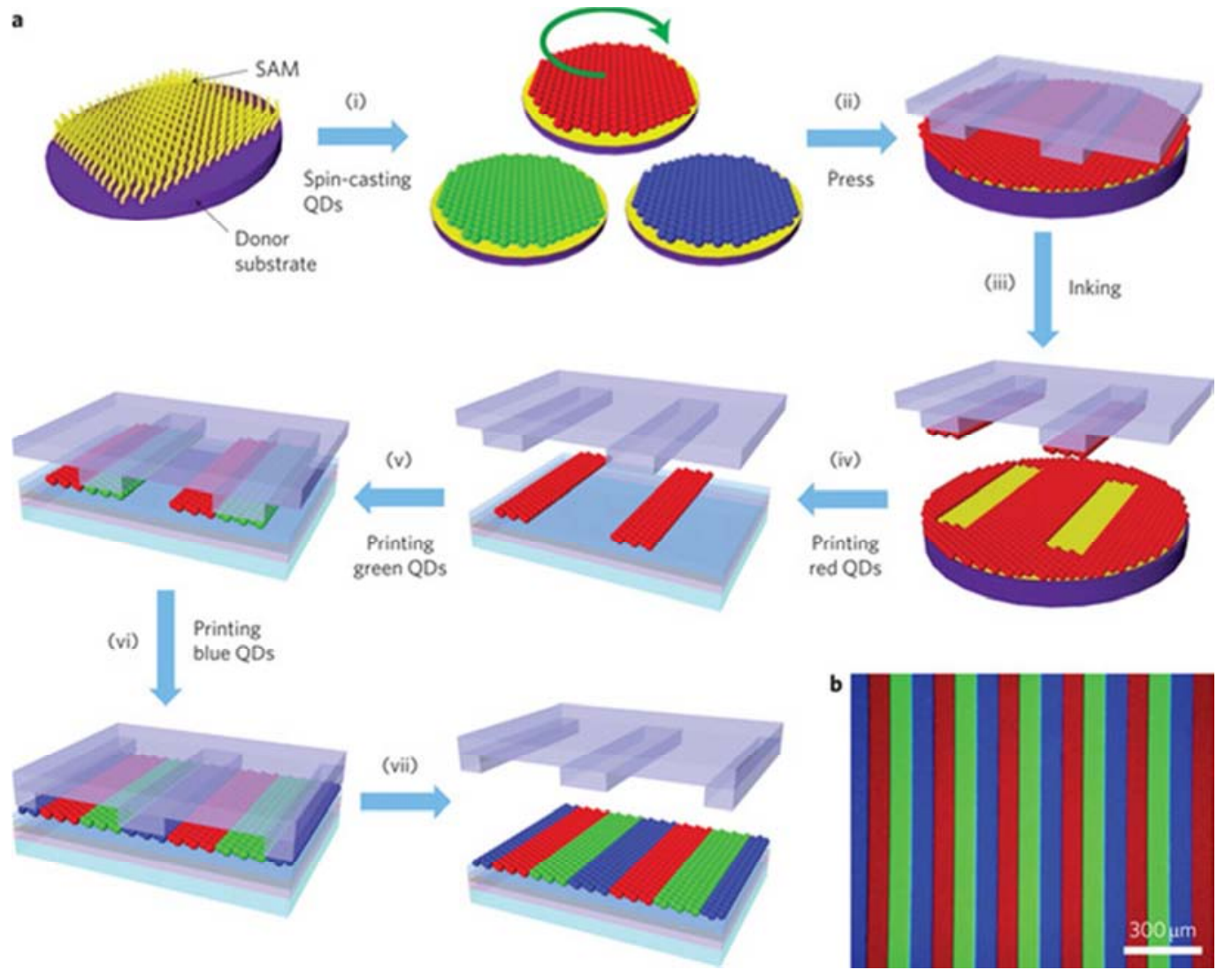

Figure 10. Quantum dot display structure (Nature).

\section{Quantum Dot Display Advantages}

(1) Pure color will deliver 30-40\% luminance efficiency advantage over organic light emitting diodes (OLEDs) at the same color point.
(2) Low power consumption QLEDs have the potential to be more than twice as power efficient as OLEDs at the same color purity.

(3) Low-cost manufacture the ability to print large-area 
QLEDs on ultra-thin flexible substrates will reduce luminaire manufacturing cost.

(4) Ultrathin, transparent, flexible form factors QLEDs will enable designers to develop new display and lighting forms not possible with existing technologies [43].

\section{Interferometry Modulator Display (IMOD)}

Interferometric modulator display (IMOD, trademarked mirasol) [44] is a technology used in electronic visual displays that can create various colors via interference of reflected light. The color is selected with an electrically switched light modulator comprising a microscopic cavity that is switched on and off using driver integrated circuits similar to those used to address liquid crystal displays (LCD). An IMOD-based reflective flat panel display includes hundreds of thousands of individual IMOD elements each a microelectromechanical systems (MEMS)-based device. In one state an IMOD subpixel reflects light at a specific wavelength, while in a second state it absorbs incident light and appears black to the viewer, using a diffraction grating effect. [45] When not being addressed, an IMOD display consumes very little power. Unlike conventional back-lit liquid crystal displays, it is clearly visible in bright ambient light such as sunlight. IMOD prototypes as of mid-2010 could emit 15 frames per second (fps), [46] and in November 2011 Qualcomm demonstrated another prototype reaching 30 fps, suitable for video playback. The recent smartwatch Qualcomm Toq features this display with 40 fps [47].

Interferometry Modulator Display Advantage

(1) Bistability: This nature inspired MEMS-based innovation is bistable. This allows nearzero power usage in situations where the display image is unchanged. This bistability derived from the inherent hysteresis of the material causes considerable power savings, especially compared to displays that continually refresh, such as LCDs.

(2) Speed: Since visible light wavelengths lie in the nanometer scale (i. e. $380 \mathrm{~nm}$ to $780 \mathrm{~nm}$ ), the deformable IMOD membrane only has to move a short distance $(\sim 100 \mathrm{~nm})$ in order to switch between two colors. This switching happens extremely fast, on the order of tens of microseconds. This is 1000 times faster than that of traditional displays. Higher switching speed directly translates to a video rate-capable display with no motion-blur effects.

(3) Robustness: In addition to microsecond switching, mirasol displays maintain their switching speed across a wide temperature range. In contrast, the switching speeds of organic liquidcrystal- based displays decrease as temperatures go into low environmental ranges.

(4) Readability: A mirasol display offers a superior contrast ratio in brightly lit environments. Qualcomm's mirasol displays offer reflectivity on the order of 60 percent and contrast ratios greater than 10: 1. By comparison, the Wall Street Journal newspaper offers a reflectivity of 60 percent and a contrast ratio of around 4: 1Reflective technology based on bio-mimetic models hold much promise. For commercial use, reflective displays they need to be viewed in both daylight and in the dark. The commercialization of this technology is thus dependent on combining the benefits of backlit Trans missive displays with the outdoor readability of reflective technologies. [48]

The advantages of IMOD displays are that they require very little power, feature excellent view ability even in bright sunlight, high readability, robust functionality, technical flexibility and mechanical durability.

Result and discussion:

One of the earliest electronic displays is the cathode ray tube (CRT), which was made commercial in 1922. The CRT consists of an electron gun that forms images by firing electrons onto a phosphor-coated screen. The earliest CRTs were monochrome and were used primarily in oscilloscopes and black and white televisions. The first commercial colour CRT was produced in 1954. CRTs were the single most popular display technology used in television sets and computer monitors for over half a century; it was not until the 2000s that LCDs began to gradually replace them. A derivative of CRTs were storage tubes, which had the ability to retain information displayed on them, unlike standard CRTs which need to be refreshed periodically. In 1968, Tektronix introduced the Direct-View Bistable Storage Tube, which went on to be widely used in oscilloscopes and computer terminals. A display is a computer output surface and projecting mechanism that shows text and often graphic images to the computer user, using a cathode ray tube (CRT), liquid crystal display (LCD), light-emitting diode, gas plasma, or other image projection technology. The way in which information, communication and entertainment is currently presented to us is quite astonishing. Thanks to the evolution of the smartphone, tablets and other mobile-based devices, as well as the ever-changing technology used in cinemas, in TV and in advertising campaigns, display technology has already penetrated our everyday lives and now this technology is set to evolve even further in the nottoo-distant future. It's been a long time coming but very soon everyone will be able to experience quality VR like never before. Various 3D headsets are being developed by a number of companies and the latest releases include the Oculus Rift and of course Sony's Project Morpheus. Combining immersive technology with motion tracking, the user will be transformed into a virtual all -encompassing environment and the potential for the tech is limitless. Modern display technologies provide higher resolution, lower power consumption, and superb optical performance. These extraordinary performing displays are cost effective and are a great fit for all different types of consumer applications. Still, when we consider the ever-growing military applications, display offerings are still limited, challenging, and costly. Display applications in the military sector are exploding. From personalized handheld devices in the field to unmanned aerial vehicles and robotics, however, standard display offerings for these applications are not 
available. So who knows what the future holds for display technologies. Additional and special modifications are a must to meet the temperature specs, environmental specs, and reliability specs. For these reasons, military grade displays are bulky, not sexy (although they may not be required to look like an Apple or a Surface), and extremely pricey. The display industry should look to develop native display panels, which can meet some of these military specifications specifically the temperature and IPC spec. Integrated Capacitive Touch with display panels would also make a good addition to military applications. For a second tier integrator this problem could actually be considered good news, as they can build products more cost effectively that may ultimately benefit the military. It's fast-moving and evergrowing. Just a few years ago we were all gathered around CRT TVs. Now we use services like Netflix to watch ondemand movies and series on our tablet. We really hope all these display technologies get perfected and become as well accepted as smartphones. The future of displays is fascinating.

\section{References}

[1] Tripathi, Mrs Alpana, and Vivek Kumar Pandey. "Effect of Television on Adolescents."

[2] Fritze, Dorian Christine Webb. "Fiber optic display systems and related methods." U. S. Patent No. 7,985,016. 26 Jul. 2011.

[3] http://www.displaymate.com/crts.html.

[4] Dictionary, A. Heritage. "The American Heritage science dictionary." (2005).

[5] Moreno, Ivan, and Ching-Cherng Sun. "Modeling the radiation pattern of LEDs." Optics express 16.3 (2008): 1808-1819.

[6] Blue, L. E. D. "Light-emitting diode explained."

[7] Alim, Jinnah Barkatul, and Tasauf Islam. Photo emission characteristics of CNT based light emitting diode. Diss. BRAC University, 2013.

[8] Narra, Prathyusha, and Donald S. Zinger. "An effective LED dimming approach." Industry Applications Conference, 2004. 39th IAS Annual Meeting. Conference Record of the 2004 IEEE. Vol. 3. IEEE, 2004.

[9] Sheng, Chieh-Wen, and Ming-Chia Chen. "A study of experience expectations of museum visitors." Tourism management 33.1 (2012): 53-60.

[10] Webster, Merriam. "Definitions-LED report a problem."

[11] Luginbuhl, Christian B., Paul A. Boley, and Donald R. Davis. "The impact of light source spectral power distribution on sky glow." Journal of Quantitative Spectroscopy and Radiative Transfer 139 (2014): 21-26.

[12] Aubé, Martin, Johanne Roby, and Miroslav Kocifaj. "Evaluating potential spectral impacts of various artificial lights on melatonin suppression, photosynthesis, and star visibility." PLoS One 8.7 (2013): e67798.

[13] https://en.wikipedia.org/wiki/Electroluminescent_display.
[14] http://www.tech-faq.com/what-is-electroluminescence.html.

[15] Heikenfeld, Jason, et al. "Review Paper: A critical review of the present and future prospects for electronic paper." Journal of the Society for Information Display 19.2 (2011): 129-156.

[16] Yadav, Shubham1 Manish. "E Paper Technology." (2016).

[17] http://www.versiondaily.com/advantages-disadvantageselectrophoretic-display/.

[18] http://money.cnn.com/2014/10/30/technology/plasma-tv/.

[19] http://www.forbes.com/sites/johnarcher/2015/11/10/oled-tvbeats-plasma-tv-in-new-public-shoot-out/\#5f688e594727.

[20] Whitaker, Jerry C., ed. The electronics handbook. Crc Press, 1996.

[21] Bagher, Askari Mohammad. "OLED Fabrication for use in Display Systems and Comparison with LCD and PLASMA."

[22] http://www.merriam-webster.com/dictionary/lcd.

[23] http://www.displaymate.com/lcds.html.

[24] "High-Performance Addressing - HPA". Computerhope.com. Retrieved 2011-01-30.

[25] "Input/Output Systems and Peripheral Devices" (PDF). utcluj.ro. 2010-11-18. Retrieved 2011-01-30.

[26] LCD Panel Technology Explained." Pchardwarehelp.com. Retrieved 2013-07-21.

[27] http://www.amorphyx.com/technology/thinfilmtransistors.

[28] http://www.allshore.com/blog/post/2012/03/22/An-Overviewof-the-Pros-and-Cons-of-TFT-Displays.aspx.

[29] Bagher, Askari Mohammad. "OLED Display Technology." Am. J. Opt. Photonics 2 (2014): 32-36.

[30] Bagher, Askari Mohammad. "Comparison of organic solar cells and inorganic solar cells." Int J Renew Sustain Energy 3 (2014): 53-58.

[31] Kinder, Marsha. "Music video and the spectator: Television, ideology and dream." Film Quarterly (1984): 2-15.

[32] Serkan Toto, "FED: Sony calls it quits, basically burying the technology as a whole", CrunchGear, 31 Mar 2009.

[33] Martyn Williams, "Canon signals end of the road for SED TV dreams", IDG News Service, 19 August 2010.

[34] Serkan Toto, "FED: Sony calls it quits, basically burying the technology as a whole", CrunchGear, 31 Mar 2009.

[35] http://www.digitimes.com/news/a20100121PD207.html.

[36] Ghrayeb, Joseph, et al. "Review of field emission display potential as a future (leap-frog) flat panel technology." AeroSense'97. International Society for Optics and Photonics, 1997.

[37] http://indiana.edu/ hightech/fpd/define.htm.

[38] German Patent 1193844 entitled "Optischer Sender fuer mindestens zwei Farbkomponeneten" was filed on October 26, 1963 by - and awarded on January 20, 1966 to - the German company Telefunken. Helmut K. V. Lotsch has explicitly been named the inventor. 
[39] Quantum-dot displays could outshine their rivals, New Scientist, 10 December 2007.

[40] Dabbousi, B. O., et al. "Electroluminescence from CdSe quantum - dot/polymer composites." Applied Physics Letters 66.11 (1995): 1316-1318.

[41] Erdem, Talha, and Hilmi Volkan Demir. "Color science of nanocrystal quantum dots for lighting and displays." Nanophotonics 2.1 (2013): 57-81.

[42] Shirasaki, Yasuhiro, et al. "Emergence of colloidal quantum-dot light-emitting technologies." Nature Photonics 7.1 (2013): 13-23.

[43] http://www.qled-info.com/introduction/.
[44] Gutherie, Audrey H., et al. "Standards for small visual displays."

[45] Watters, Ethan (June 12, 2007). "Product design, nature's way". CNNMoney. Cable News Network.

[46] Gabryel (July 7, 2010). "Qualcomm mirasol launch its color eReader". GeeksHive blog.

[47] Simonite, Tom (November 15, 2011). "E-Reader Display Shows Vibrant Color Video". Technology Review. Massachusetts Institute of Technology.

[48] Miles, M., et al. "Digital Paper ${ }^{\mathrm{TM}}$ for reflective displays." Journal of the Society for Information Display 11.1 (2003): 209-215. 